\title{
New method of determination of the tool rake angle on the basis of the crack angle of the specimen in tensile tests and numerical simulations
}

\author{
L. Kukielka ${ }^{1}$, J. Chodor $^{1}$ \& B. Storch ${ }^{2}$ \\ ${ }^{I}$ Department of Mechanical Engineering, \\ Koszalin University of Technology, Poland \\ ${ }^{2}$ Department of Processes Monitoring, \\ Koszalin University of Technology, Poland
}

\begin{abstract}
Turning is a very complicated technological process. To increase the quality of the product and minimize the cost of turning, we should know the physical phenomena that exist during the process. In some of the literature the thermo-mechanical models and other dependencies between the shape of the tool and the shape of the chip are presented. This paper is a continuation of previous issues and focuses on proper determination of active cutter geometry. A new method of determination of tool rake angle $\gamma$ in two steps is presented. In the first step the basic angle $\gamma$ is determined on the basis of the crack angle of the specimen in a tensile test. In the second step the optimal angle $\gamma$ is determined using numerical simulations. The influence of the cutter geometry in the active part and the tool rake angle $\gamma$ on the states of strain and stress in the surface layer during turning is explained. The phenomena on a typical incremental step were described using a step-by-step incremental procedure, with an updated Lagrangian formulation. The turning process is considered as a geometrical and physical non-linear initial and boundary problem. The finite element method (FEM) and the dynamic explicit method (DEM) were used to obtain the solution. The application was developed in the ANSYS/LS-DYNA system, which makes possible a complex time analysis of the physical phenomena: states of displacements, strains and stresses. Numerical computations of the strain have been conducted with the use of methodology, which requires a proper definition of the contact zone, without the necessity to introduce boundary conditions. Examples of calculations are presented.
\end{abstract}

Keywords: turning, chip creation, tensile test, yield stress, FEM, numerical analysis, state of strain, state of stress. 


\section{Introduction}

In order to improve metal cutting processes, e.g. lower the part cost, it is necessary to model metal cutting processes at the system level. A necessary requirement of such is the ability to model interactions at the tool-chip interface and thus, predict cutter performance. Some model of testing for model development, either material, machining, or both, is required for all. However, the ability to model cutting tool performance with a minimum amount of testing is of great value, reducing costly processes and tooling iterations.

The process of the chip creation proves to have a substantial influence on the turning process, together with the geometrical and kinematics' dependencies in the contact zone of the cutter - the object machined. It has a significant impact on the wear of the cutter, the value of the components of the turning force, the temperature and the quality of the surface machined.

During the turning process, there occur large and fast plastic strains, which occur only in the part of the object machined. Under the influence of these strains in the material, its physical properties are subject to change: the hardness and strength increase. There occurs the so-called consolidation phenomenon of the material. The geometry of the zone of the chip creation during machining allows one to accept an assumption that in the area in question there is a plate state of strains.

For the correct modelling and analysis of the turning process, knowledge of the course of the physical phenomena occurring in the machining zone in real conditions (i.e. the geometry of the cutter and the technological parameters) proves to be necessary. For this purpose, an analysis of the process of turning with a cutter was conducted. The object is considered as the elastic/visco-plastic body and it rotates with the rate of turning of $\omega$ around its own axis (Fig. 1).

In papers $[1,2]$, a thermal and mechanic model of the process of the grain displacement on the elastic/visco-plastic body was developed and the distribution of temperatures were determined together with the intensities of strain in the material machined in the initial chip creation phase.

This paper shows how to properly create the surface layer of the product. The finite element method (FEM) is used to calculate chip geometry, its formation and separation from the body. A new method of determination of the tool rake angle $\gamma$ in two steps is presented. Initially, the basic tool rake angle $\gamma$ was determined from a tensile test. Then, using numerical simulation, the optimal angle $\gamma$ was established. This ensures the best durability of the cutter, i.e. the lowest power of material deformation. For this case the states of stress and strains in the surface layer of the object at any time of the process were calculated.

\section{Basic cutter rake angel}

\subsection{Method of determination}

An analogy of physical phenomena was used during the turning and tensile test. According to the literature [6], necking, which is made in specimen in the tensile 


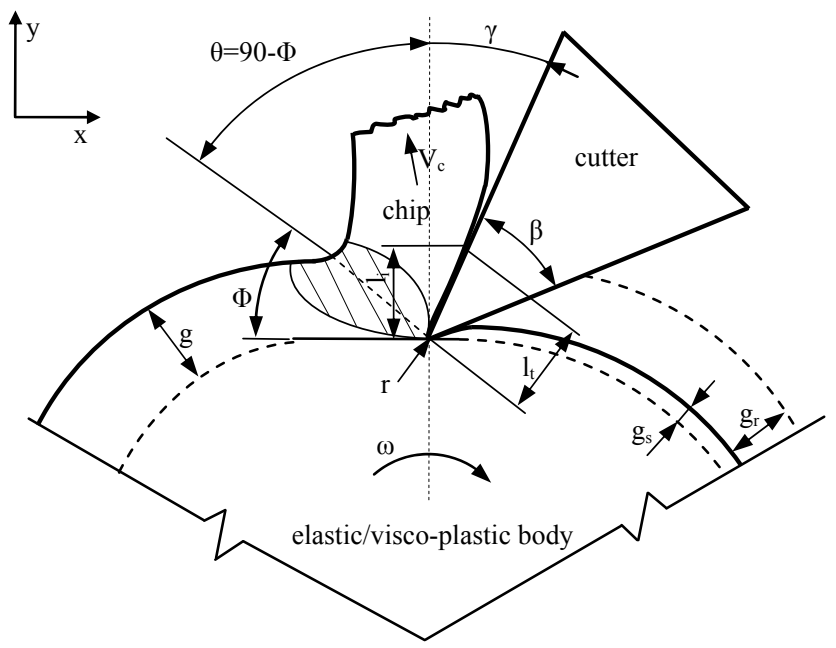

Figure 1: Diagram of the considered issue of the elastic/visco-plastic body with rate of turning $\omega$ using a $2 \mathrm{D}$ model ( $\mathrm{g}$ is the depth of cut, $\mathrm{g}_{\mathrm{r}}$ is the real depth of cut, $g_{s}$ is the elastic deformation of material, $\beta$ is the cutter angle in active part, $r$ is the corner radius, $\omega$ is the rate of turning, $\Phi$ is the cutting angle, $\gamma$ is the tool rake angle, $V_{c}$ is the chip velocity).

a)

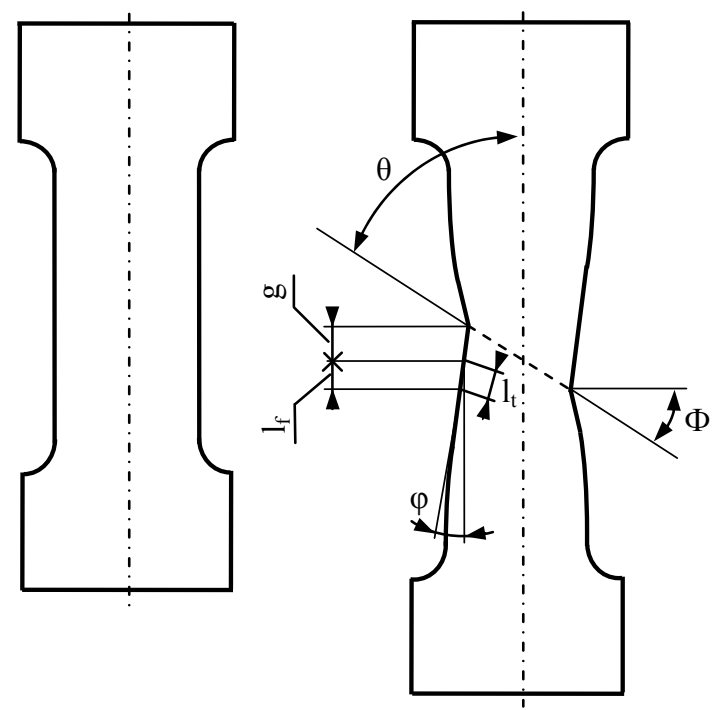

Figure 2: View of specimen: a) before tensile test, b) after tensile test. 
test (Fig. 2), is similar for every kind of material (crack angle $\Phi \sim 45^{\circ}$ ). Many numerical calculations and their verification in real conditions do not overlap with the above statement.

In this section the first step to determinate a basic tool rake angle $\gamma$ is shown. An analogy of physical phenomena was used during the turning and tensile test. The cutting angle $\Phi$ and crack angle $\Phi$ are similar. During the tensile test, the elongation of specimen and necking angle $\varphi$ depend on the plasticity of the material. The tool rake angle $\gamma$, which is necessary for separating the material, can be described using the necking angle $\varphi$ and the parameter of elongation $\xi$ defined as (Fig. 2):

$$
\xi=\left(l_{t}-l_{i}\right) / l_{i}
$$

From Fig. 1, we have:

$$
\cos \gamma=l_{i} / l_{t}
$$

and from Fig. 2:

$$
\cos \varphi=l_{\mathrm{f}} / \mathrm{l}_{\mathrm{t}} .
$$

Using $1_{\mathrm{f}}$ determined from formula (2) and (3), we obtain:

$$
\cos \gamma=\mathrm{l}_{\mathrm{i}} \cos \varphi / \mathrm{l}_{\mathrm{f}},
$$

and then the value of the rake:

$$
\left.\gamma=\arccos \left(\mathrm{l}_{\mathrm{i}} \cdot \cos \varphi / \mathrm{l}_{\mathrm{f}}\right)=\arccos \left[\mathrm{l}_{\mathrm{t}} \cdot \cos \varphi\right) / \mathrm{l}_{\mathrm{f}} \cdot(\xi+1)\right]
$$

The necessary angle $\varphi$ and parameter $\xi$ are determined from the tensile test.

\subsection{Experimental results}

Tensile tests were made on a testing machine ZD20 $(200 \mathrm{kN})$ to determine the angle $\varphi$ and parameter $\xi$, required to determine the basic tool rake angle $\gamma$.

Many experiments were carried out on four different materials: stainless steel 303, 4340 steel, AISI 1042 steel and titanium alloy Ti6AI4V. Fig. 3 shows the sample after the tensile test on universal testing machine ZD 20.

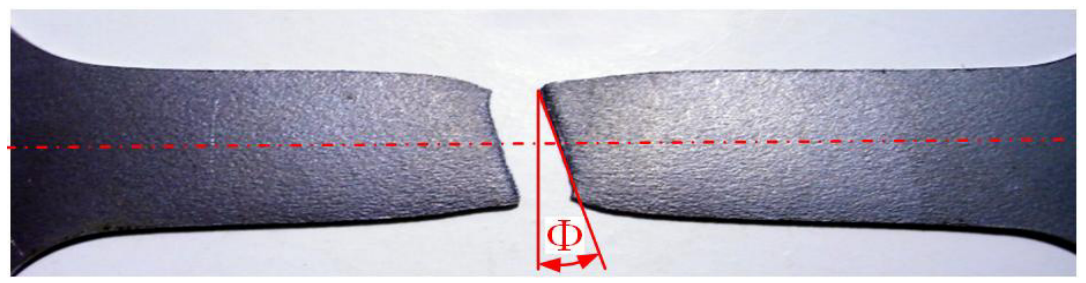

Figure 3: $\quad$ View of specimen after tensile test with marked crack angle $\Phi$ for stainless steel 303. 
After research we obtained the following tool rake $\gamma$ angles: stainless steel 303 is $\gamma=46^{\circ}$, AISI 4340 steel is $\gamma=40^{\circ}, 1042$ steel is $\gamma=44^{\circ}$, and titanium alloy Ti6Al4V is $\gamma=33^{\circ}$. Then, using the above cutters, the turning process was carried out. Experiments were made on the same machining centre - NEF400 (FANUC MANUAL GUIDE) with traverse $\mathrm{f}=0.1$ [mm] and depth of cut $\mathrm{g}=1.9[\mathrm{~mm}]$. The velocity of machining was different and depended on the type of material. The shapes of the chips after machining were different and were characteristic for every type of material. The original tool, used for comparison, had the same tool rake angle $\gamma=-7^{0}$.

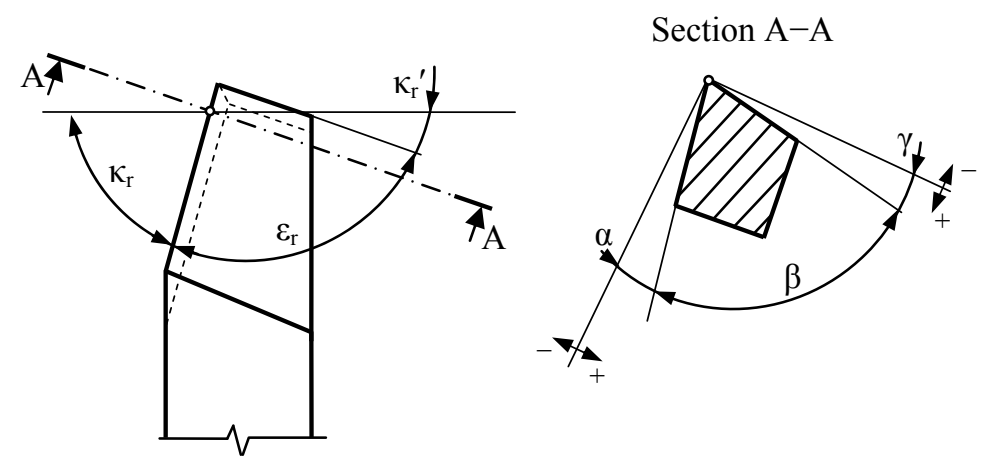

Figure 4: $\quad$ Main angles of the cutter [7].

Experiment 1: Material machined - stainless steel 303.

Parameters of new geometry tool: $\gamma=46^{\circ}, \kappa_{r}=46^{\circ}$, and width of chip: $X=0,127[\mathrm{~mm}]$. The main angles of the cutter are shown in Fig. 4, where $\varepsilon_{\mathrm{r}}$ is the apex angle of the cutter and $\kappa_{\mathrm{r}}$ and $\kappa_{\mathrm{r}}^{\prime}$ are the main and auxiliary tool cutting edge angles, respectively.

Experiment 2: Material machined - AISI 4340 steel.

Parameters of new geometry tool: $\gamma=40^{\circ}, \kappa_{r}=40^{\circ}$ and $X=0,254[\mathrm{~mm}]$.

Five experiments were carried out using the base geometry of the tool and four experiments were carried out with the new geometry of the cutter. The tool with the original geometry allowed one to machine about 504 [mm] of material, whereas the new geometry tool allowed one to machine about $820[\mathrm{~mm}]$ of material.

Experiment 3: Material machined - 1042 steel.

Parameters of new geometry tool: $\gamma=44^{0}, \kappa_{r}=44^{0}$ and $X=0,127[\mathrm{~mm}]$.

Four experiments were carried out using the base geometry of the tool and four experiments were carried out with the new geometry of the cutter. The durability of the new geometry tool was much higher than the old one.

Experiment 4: Material machined - titanium alloy Ti6AI4V.

Parameters of new geometry tool: $\gamma=33^{\circ}, \kappa_{r}=33^{\circ}$ and $X=0,127[\mathrm{~mm}]$. 


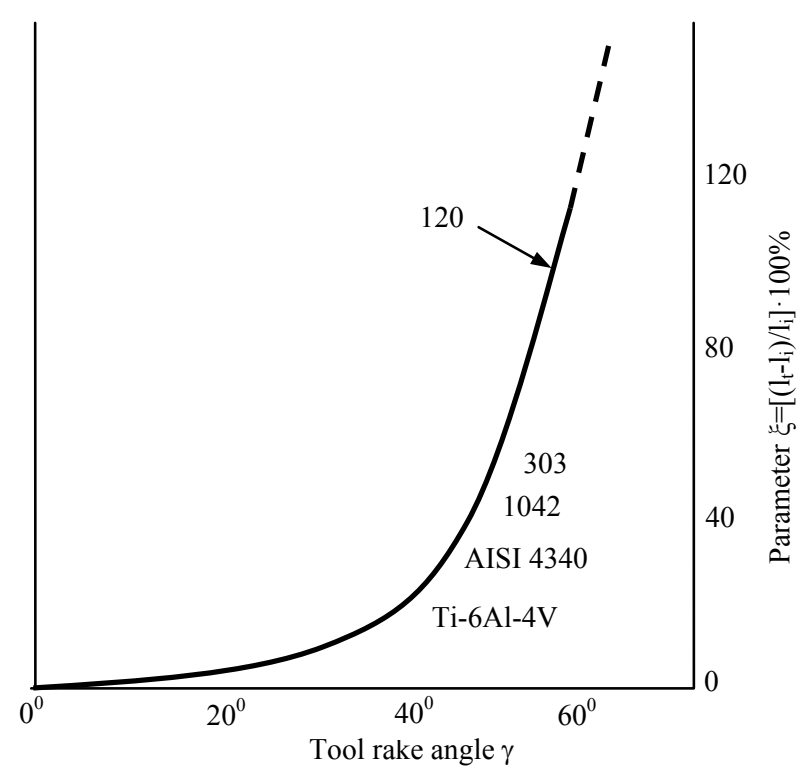

Figure 5: Dependence between tool rake angle $\gamma$ and decreasing parameter of machining resistance $\xi$.

Three experiments were carried out using the base geometry of the tool and four experiments were carried out with the new geometry of the cutter. The durability of the new geometry tool was higher than the old one.

Fig. 5 shows the visual results of the experiments. Curve number 120 shows dependence between tool rake angle $\gamma$ and parameter $\xi$. The angle changes between $0^{\circ}$ and $24^{\circ}$ for fragile materials (e.g. cast iron) and for materials that have unit elongation of about $10 \%$ (e.g. constructional steels, stainless steels, non-ferrous metals, aluminium, titanium).

The optimal tool rake angle $\gamma$ is in situation when the turning power, which is dependent (for set-up turning feed and velocity) on the deformation power of the turned material, is low. If the turning power is lower than the durability of the cutter, the tool rake angle is larger. The static tensile test takes place during low strain rates of about $\dot{\varepsilon}_{\mathrm{i}}=10^{-4} \mathrm{~s}^{-1}$, but real strain rates of material during turning are much higher and equal about $\dot{\varepsilon}_{\mathrm{i}}=10^{4} \div 10^{5} \mathrm{~s}^{-1}$, so modification of angle $\gamma$ seems to be necessary. To solve this problem and to determine the optimal angle $\gamma$, numerical simulations of the turning process were conducted.

\section{Numerical calculations}

\subsection{Numerical algorithm}

Turning is considered as a geometrical and physical nonlinear initial and boundary problem. The analytic solution of this problem, such as the 
determination of states of deformations and stresses in any moment of duration of the process, is impossible. Therefore this problem was solved by the FEM. An application was developed in the Ansys/LS-Dyna programme, which makes a complex time analysis of the states of deformation (displacements and strain) and stress in the surface layer of the object at/after turning possible.

For the purpose of the solution of the problem, the Dynamic Explicit Method (DEM), also known as the Method of Central Differences, was used. In this method, the equation that describes the movement and deformation of the object investigated on the typical step time, in updated Lagrangian formulation, has the following form:

$$
\mathbf{M} \cdot \Delta \ddot{\mathbf{r}}+\mathbf{C}_{\mathrm{T}} \cdot \Delta \dot{\mathbf{r}}+\left(\mathbf{K}_{\mathrm{T}}+\Delta \mathbf{K}_{\mathrm{T}}\right) \cdot \Delta \mathbf{r}=\Delta \mathbf{R}_{\mathrm{T}}+\Delta \mathbf{F}+\mathbf{F}_{\mathrm{T}}
$$

where $\mathbf{M}$ is the global system-mass matrix at time $\mathrm{t}, \mathbf{C}_{\mathrm{T}}$ is the global systemdamping matrix at time $t, \mathbf{K}_{\mathrm{T}}$ is the global system stiffness matrix at time $\mathrm{t}, \Delta \mathbf{K}_{\mathrm{T}}$ is the global system stiffness-increment matrix at the step $\Delta \mathrm{t}, \mathbf{F}_{\mathrm{T}}$ is the global system internal and external load vector at time $\mathrm{t}, \Delta \mathbf{F}$ is the global system internal load increment vector at the step $\Delta \mathrm{t}, \Delta \mathbf{R}_{\mathrm{T}}$ is the global system external load increment vector at step time $\Delta \mathrm{t}, \Delta \mathbf{r}$ is the global system displacement increment vector at step time $\Delta \mathrm{t}, \Delta \dot{\mathbf{r}}$ is the global system velocity increment vector at step time $\Delta \mathrm{t}$ and $\Delta \ddot{\mathbf{r}}$ is the global system acceleration increment vector at step time $\Delta \mathrm{t}$. This equation is not solvable due to the number of unknowns exceeding the number of equations. An approximation by the central difference method has been applied to express $\dot{\mathbf{r}}$ and $\ddot{\mathbf{r}}$ vectors using the displacement vectors at moments: $\mathrm{t}-\Delta \mathrm{t}, \mathrm{t}, \mathrm{t}+\Delta \mathrm{t}$ :

$$
\dot{\mathbf{r}}^{\mathrm{t}}=\frac{1}{2 \Delta \mathrm{t}} \cdot\left(\mathbf{r}^{\dot{\mathrm{t}}+\Delta \mathrm{t}}-\mathbf{r}^{\mathrm{t}-\Delta \mathrm{t}}\right) \text {, and } \ddot{\mathbf{r}^{\mathrm{t}}}=\frac{1}{\Delta \mathrm{t}^{2}} \cdot\left(\mathbf{r}^{\mathrm{t}+\Delta \mathrm{t}}-2 \mathbf{r}^{\mathrm{t}}+\mathbf{r}^{\mathrm{t}-\Delta \mathrm{t}}\right) \text {. }
$$

It was accepted in the simulations that the cutter (Fig. 6) is a non-deformable body, but it can also be an elastic body for precise calculations, while the object is an elastic/visco-plastic body described with the aid of the Cowper-Symonds model. The Huber-Mises-Hencky plasticity model is used together with the associated flow right. The model takes into consideration the line-isotropic $(\eta=1)$ kinematic $(\eta=0)$ or mixed $(0<\eta<1)$ plastic hardening, as well as the influence of the intensity of the plastic strain rate, according to the involution dependence:

$$
\sigma_{\mathrm{Y}}=\left(\sigma_{0}+\eta \cdot \mathrm{E}_{\mathrm{tan}} \cdot \varepsilon_{\mathrm{i}}^{(\mathrm{p})}\right) \cdot\left[1+\left(\dot{\varepsilon}_{\mathrm{i}}^{(\mathrm{p})} / \mathrm{C}\right]^{\mathrm{m}} \quad[\mathrm{MPa}]\right.
$$

where $\sigma_{\mathrm{Y}}$ is the yield stress, $\sigma_{0}[M P a]$ is the initial yield stress point, $\varepsilon_{\mathrm{i}}^{(\mathrm{p})}[-], \dot{\varepsilon}_{\mathrm{i}}^{(\mathrm{p})}\left[\mathrm{s}^{-1}\right]$ is the intensity of true strain and plastic true strain rate respectively, $\mathrm{C}\left[\mathrm{s}^{-1}\right]$ is the material parameter to determine the influence of the intensity of the plastic strain rate, $\mathrm{m}=1 / \mathrm{P}$ is the material constant determining the sensitiveness of material on the plastic strain rate and $E_{\tan }=E_{T} E /\left(E-E_{T}\right)$ is the material parameter dependent on the module of plastic hardening $\mathrm{E}_{\mathrm{T}}=$ 
$\partial \sigma_{\mathrm{Y}} / \partial \varepsilon_{\mathrm{i}}^{(\mathrm{p})}$ and of Young's elasticity module E. The parameters used in numerical calculations depended on the material, as each one was different.

\subsection{Optimal tool rake angle}

In order to determine the optimal tool rake angle $\gamma$ for the chosen material, numerical simulations were conducted. Angle $\gamma$ was changed every $0^{0} 30^{\prime}$ (increasing and decreasing), starting from angle $\gamma$, which was determined from the tensile test (section 2).

The chosen results of the numerical simulations of the states of stress and strain during turning stainless steel 303 with $\beta=50^{\circ}$ and $\gamma=20^{\circ}$ are shown in Fig. 6.

a)

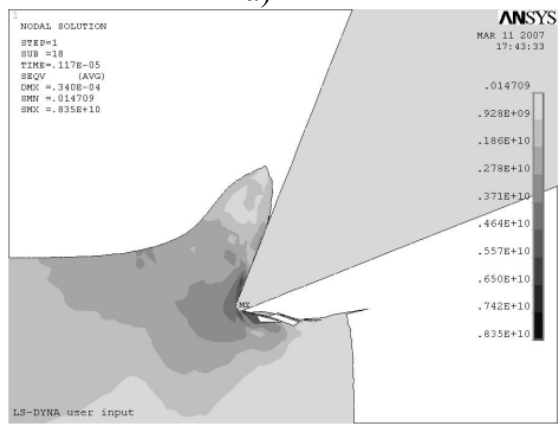

c)

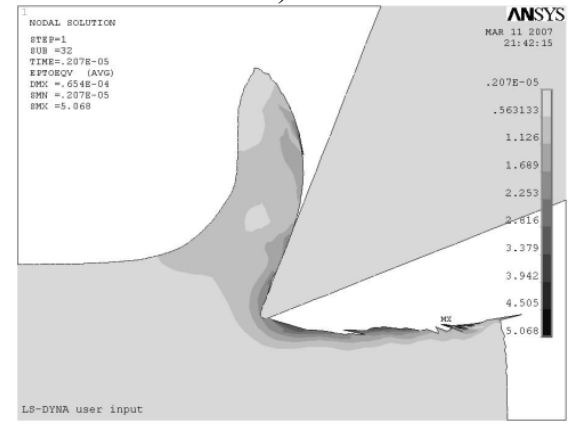

b)

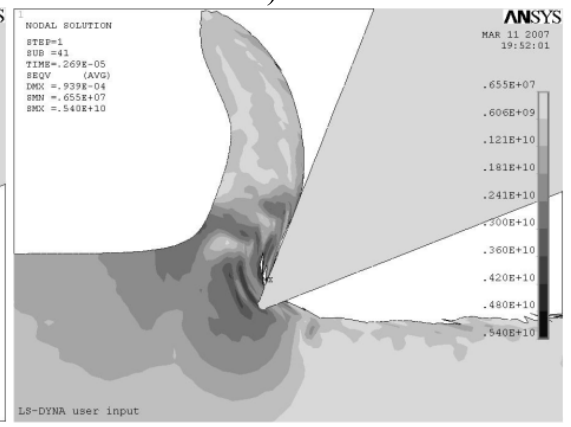

d)

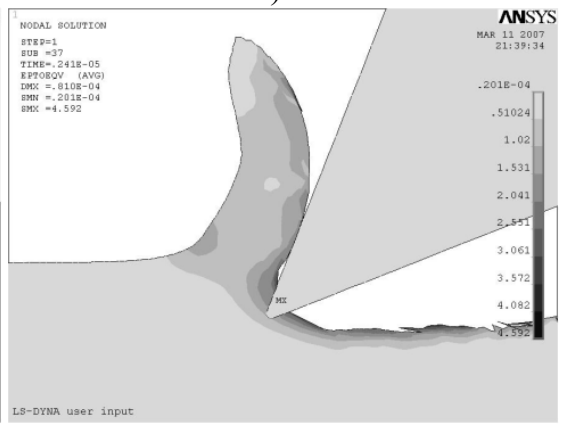

Figure 6: View of 2D turning with example chip shapes and marked stress $(\mathrm{a}, \mathrm{b})$ and strain $(\mathrm{c}, \mathrm{d})$ distribution.

In Fig. 6 we can observe high values of stress intensities (b), especially in the plane of shear and in the contact zone of the cutter with the chip. The high value of strain is in the surface layer of material machined (d).

Fig. 7 shows turning of the AISI 4340 steel with $\beta=70^{\circ}$ and $\gamma=10^{\circ}$. We can notice the strip chip, which is curling in the direction of the foundation. This is characteristic for high velocity machining. 
a)

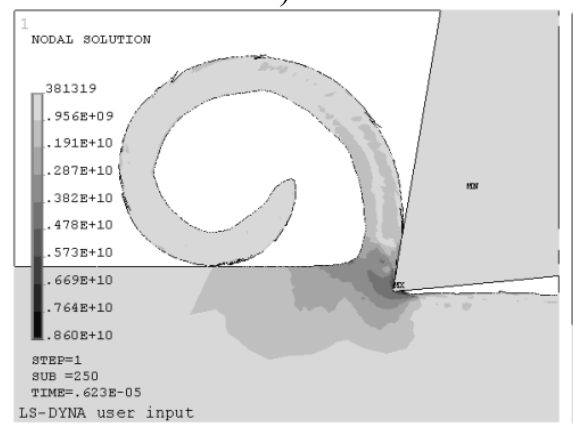

b)

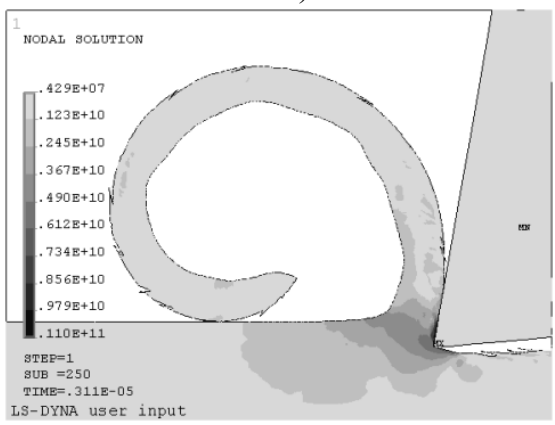

Figure 7: View of 2D turning with example chip shapes and marked stress distribution $(\mathrm{a}, \mathrm{b})$.

a)

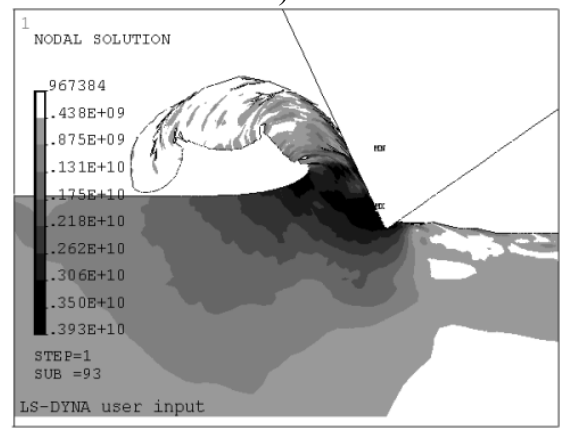

b)

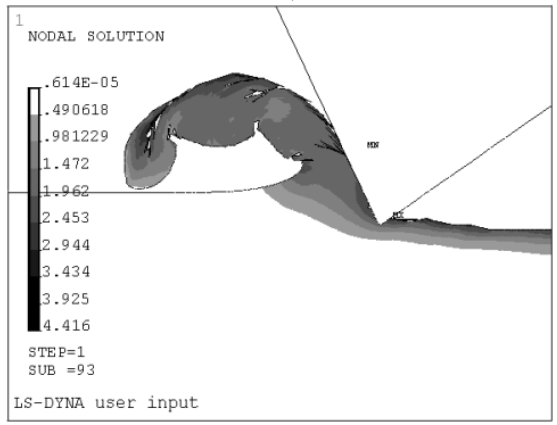

Figure 8: $\quad$ View of 2D turning with example chip shapes and marked stress (a) and strain (b) distribution.

In Fig. 8 we can observe the turning process of 1042 steel with $\beta=80^{\circ}$ and $\gamma=-25^{\circ}$. The chip that is created is a step chip. The maximum value of stress is in the plane of shear and equals about $\varepsilon=3900$ [MPa].

From the above figures it can be seen that the smaller value of angel $\gamma$ causes much better strain distribution in the material machined, especially in the surface layer. However, the growth of angel $\gamma$ causes much less machining resistance and stress distribution.

\section{Conclusions}

Experiments shows us that the real cutting angle changes between $33^{\circ}$ and $46^{\circ}$ or between $39^{\circ}$ and $53^{\circ}$. The geometry of the tool that is presented in this paper is the result of many numerical calculations and experiments in real conditions. It allows machining materials with a minimum loss of energy, minimum heat transfer and much greater strength of the tool material. 
An application of modern numerical methods and computing systems allows an analysis of the complex physical phenomena occurring in the process under investigation. The application developed in the ANSYS/LS-DYNA system enables a time analysis of the process of machining with a cutter, with the consideration of the changeability of the cutter's apex angle and the tool rake angle.

The distribution of stresses and strains obtained for different cutter geometries and action angles, in particular the phases of the deformation process, can be made use of while designing machining: making a selection of the machining conditions and their optimisation in the aspect of the technological quality of the product.

The material flashes obtained before the cutting edge and the shape of the chips are similar to the results of experiential investigations, confirming the justifiability of the use of computer simulations and their reliability.

\section{References}

[1] Kukielka L., Kustra J.: Numerical analysis of thermal phenomena and deformations in processing zone in the centreless continuous grinding process. Surface Treatment VI Computation Methods and Experimental Measurements for Surface Treatment Effects. Ed. C.A. Brebbia, J.T.M. de Hosson, S-I. Nishida WIT Press, Southampton, Boston, 2003, pp.109-118.

[2] Kukielka L., Kustra J., Kukielka K.: Numerical analysis of states of strain and stress of material during machining with a single abrasive grain. Computer Methods and Experimental Measurements for Surface Effects and Contact Mechanics VII. WIT Press Southampton, Boston 2005, pp. 57-66.

[3] Kukielka L., Chodor J.: Numerical analysis of the influence of abrasive grain geometry and cutting angle on states of strain and stress in the surface layer of object. Surface/Contact 2007 Conference, Wessex Institute, Ed. J.T.M. De Hosson, C.A. Brebbia, S-I.Nishida, WIT Press Southampton, Southampton 2007, UK, pp.183-193.

[4] Kukielka L., Chodor J.: Numerical analysis of chip formation during machining for different value of failure strain. PAMM, Volume 7, Issue 1, p. 4030031-4030032. Zürich 2007, Switzerland.

[5] Kukielka L. Chodor J.: Numerical analysis of micromachining of C45 steel with single abrasive grain, 79th Annual Meeting of the International Association of Applied Mathematics and Mechanics, Bremen 2008, Germany.

[6] Mundy William L.: Cutting tool for the continuous machining of metals and the method of making same, O'Fallon Investment. Co., Express Information, Moscow 1975.

[7] Storch B.: Phenomena on cutting edge and monitoring of surface roughness after one-egde machining. Monograph of Faculty Engineering, $\mathrm{Nr}$ 124, Publisher of Koszalin University of Technology. 